\title{
Performance Evaluation of an Energy Efficient RRM strategy in Heterogeneous Cellular Networks
}

\author{
Pierpaolo Piunti, Marco Dolfi, Simone Morosi, Sara Jayousi, Enrico Del Re \\ University of Florence - CNIT, ITALY \\ Email: pierpaolo.piunti@unifi.it, simone.morosi@unifi.it, sara.jayousi@cnit.it, enrico.delre@unifi.it
}

\begin{abstract}
The objective of increasing the Energy Efficiency of communication systems must cope with a sustained growth of the market, a greater penetration of broadband services in everyday life, higher data rate services and a large increase of the number of mobile subscriptions. In this scenario, a particular attention has to be devoted to energy saving strategies that may also contribute to the reduction of greenhouse gas emissions. The goal of an effective energy saving strategy is to reduce the power consumption without introducing any significant decrease in the network and system performance. In this paper we present the complete performance evaluation of a previously proposed strategy: particularly, the considered joint Radio Resource Management (RRM) strategy is based on the use of the cell sleep mode and the reduction of the maximum transmission power at the base station; the exponential smoothing technique is applied to forecast traffic in a given area considering daily and weekly variations. A detailed evaluation of system performance is done with reference to the no energy saving case. Results show the effectiveness of the propose strategy in heterogeneous networks (Het-Nets) where cells of different sizes effectively coexist.
\end{abstract}

\section{INTRODUCTION}

Since ICT industry is accounted for the $2 \%$ of global $\mathrm{CO}_{2}$ emissions [1] and the forecast for the footprint of ICT related activities gives a worrying threefold increase for 2020, the energy efficiency is currently the focus of multidisciplinary studies. Regarding the communication systems, these activities must cope with the expected scenarios for the upcoming years, namely a sustained growth of the market, a greater penetration of broadband services in everyday life, higher data rate services and a large increase of the number of mobile subscriptions [2]. As far as the mobile and wireless communications are concerned, it is generally known that the major contribution to energy consumption is due to the access network [3] [4]: this problem has been recently dealt with by many research groups [5]-[10]. In particular, as correctly pointed out in [11], a considerable amount of the available power is consumed by the base stations, regardless the actual traffic load: as a result, the energy saving strategies must take into account fluctuations of traffic during the day. This goal can be pursued by both energy efficient radio resource management (RRM) techniques and proper deployment strategies, with no coverage, capacity and performance reduction. Moreover, the proposed strategies should also be taylored to the proposed architectures for the future mobile systems such as the heterogeneous networks (Het-Nets) where cells of different sizes effectively coexist. This paper evaluates the performance of an energy efficient RRM strategy based on the use of the cell sleep mode [12], [13] and the adaptation of the maximum transmission power at the base station (BS) [14], [15]: while the sleep mode allows to deactivate the radio stage of the BS equipment, the adaptation of the transmission power guarantees the area coverage. In [16] an energy saving strategy combining these aspects is proposed in a scenario with macro and micro base stations: besides, the joint strategy is driven by the use of a traffic forecast. The use of the forecast for energy efficiency maximisation has been already considered in [17] and [18]. As highlighted in [18], the forecast approach requires a lower number of switch on/off operations with respect to the procedure based on istantaneous traffic measurements; as a result, the signaling traffic and handover operations are also reduced. Since the implementation of an energy saving strategy should not introduce any significant decreasing in the network and system performance, this work extends the results presented in [16] by introducing a detailed evaluation of the users' quality of service (QoS) based on simulations done by a discrete event simulator (NS-3 ${ }^{1}$ ). The outputs of the evaluation show that the proposed energy efficient solution performs similarly to the reference case where the network always provides the peak traffic capacity. The paper is organised as follows. In Section II the system model and the main considered metrics are described. In Section III the energy saving approaches and the proposed solution are presented. In Section IV the results are discussed and Section $\mathrm{V}$ concludes the work.

\section{System Model}

An LTE-based network of two different layers of hexagonal cells is considered, as shown in Fig. 1. The first layer is composed of a set of 19 macro cells where a BS is placed in the center of each cell providing an omnidirectional coverage. The second layer (overlapped to the macro cells coverage area) is formed by 54 micro cells whose BSs, equipped with an omnidirectional antenna, are placed in the vertices of each first layer macro cell. While the first layer of cells aims at providing a global coverage of the selected area, the second one is considered for capacity enhancement. This deployment scheme represents an effective solution in order to fulfil the users' coverage and capacity requirements in a heterogeneous cellular network [5]: basically, while the macro BSs provide the coverage, the capacity request in the cell edge areas is

\footnotetext{
${ }^{1}$ http://www.nsnam.org
} 
TABLE I

SYSTEM PARAMETERS

\begin{tabular}{lr}
\hline \hline Basic LTE system parameters & \\
Carrier frequency & \\
Bandwidth & $2 \mathrm{GHz}$ \\
Available PRBs & FDD $10+10 \mathrm{MHz}$ \\
\hline Base stations parameters & 50 \\
macro BS transmission power & $20 \mathrm{~W}$ \\
micro BS transmission power & $5 \mathrm{~W}$ \\
antenna pattern & omnidirectional \\
macro BS intersite distance & $1000 \mathrm{~m}$ \\
micro BS intersite distance & $500 \mathrm{~m}$ \\
\hline Link budget parameters & \\
macro BS-UE pathloss & \\
micro BS-UE pathloss & $128.1+37.6 \log (d)$ \\
noise PSD & $140.7+36.7 \log (d)$ \\
UE receiver sensitivity & $-174 \mathrm{dBm} / \mathrm{Hz}$ \\
\hline Other parameters & $-90 \mathrm{dBm}$ \\
Datarate target & \\
Scheduling & $500 \mathrm{kbps}$ \\
\hline \hline
\end{tabular}

TABLE II

POWER CONSUMPTION MODEL PARAMETERS

\begin{tabular}{lccc}
\hline \hline Bs type & a & b & c \\
Macro BS & 4.7 & $130 \mathrm{~W}$ & $75 \mathrm{~W}$ \\
Micro BS & 2.6 & $56 \mathrm{~W}$ & $39 \mathrm{~W}$ \\
\hline
\end{tabular}

guaranteed by the micro BSs. We assume that both the macro and the micro BSs are e-NodeBs which resort to the LTE air interface. According to [19] the parameters to model such systems are the ones reported in Table I. The average capacity of each cell is evaluated according to the following formula:

$$
C_{a v g}=\frac{1}{S} \int_{S} W \log _{2}\left(1+P_{t x} \cdot \Gamma(s)\right) d s
$$

where $W$ is the system bandwidth, $P_{t x}$ is the power which is transmitted by the $\mathrm{BS}$ and $\Gamma(s)$ is a function that takes into account the effects of pathloss, interference and noise by varying the position of the receiver with respect to the base station. As for the power consumption of each cell, according to [20] the following power model is considered.

$$
P_{c}= \begin{cases}a \cdot P_{t x}+b, & \text { if } \mathrm{BS} \text { is } \mathrm{ON} \\ c, & \text { if } \mathrm{BS} \text { is OFF }\end{cases}
$$

In particular the value of $a, b$ and $c$ indicate respectively the variable power consumption, the fixed power consumption when the base station is active and the fixed power consumption when the base station is on sleep mode. Such values are set as described in Table II for a typical LTE system [20]. Details on the adopted traffic generation models are reported in Section III.

\section{The Forecasting BASED ENERGy SAVING STRATEGY}

In this section the proposed forecasting-based energy saving solution is described, which includes micro cell ON/OFF switching and macro cell power adaptation on traffic conditions, highlighting the adopted traffic models and the energy management algorithm.

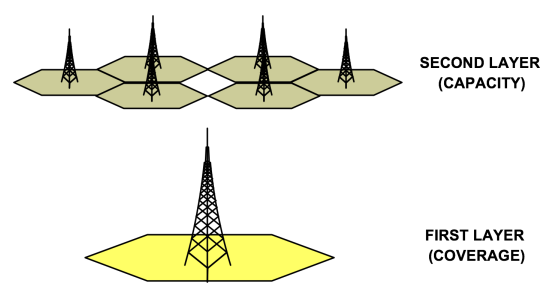

(a) Hierarchical cell structure



(b) Deployment

Fig. 1. Considered scenario

\section{A. Traffic Generation, Management and Forecasting Models}

A set of active users is considered in the interested area in order to generate traffic. Each user requests a constant bitrate datastream and the quality of the link is assumed to be acceptable if datarate is greater than a target value. Users are served by macro and micro base stations in a time sharing way and each one can be connected to only one base station at a time. To provide equal throughput to all users connected to a BS, a Blind Throughput Average scheduler is adopted [21]. This scheduling solution works by compensating a weak channel quality by a larger amount of bandwidth. In [20] the variations of data traffic during the day are modeled with a daily pattern that considers the percentage of active users during the day with respect to the global number of subscribers in a certain area. In this work the average number of simultaneous users at busy hour has been fixed and for the other hours it is calculated following this pattern. It is assumed that the number of simultaneous users follows a Poisson distribution with a different mean at each hour and that such users are uniformly distributed in the considered area. Since the effectiveness of a solution based on the joint micro cell sleep mode activation and macro cell power adaptation relies on the BSs' knowledge of the amount of the instantaneous traffic requests at a given time, a suitable traffic forecast technique is considered. The use of forecast in cellular network is justified by the correlation between traffic pattern and the hours of the day when it is observed. In [16] the traffic prediction considered in this work is presented. Such technique, namely the Holt-Winter's forecasting technique (HW) [22], is an extension of the exponential smoothing strategy which is based on a recursive scheme according to which the forecast is updated at each observation of the considered phenomena. Exponential smoothing performs the forecast of the future values of a time series starting from past values, which 
are properly weighted in so that the recent values are more important than to the older ones. H-W extends exponential smoothing introducing trend and seasonal components.

\section{B. The Proposed Strategy}

A cellular network is typically designed to fulfil the user traffic requirements at busy hours. Therefore, to reduce the power consumption, it is worth adapting the availability of the radio resources to the variations of the traffic requests during the day: this choice permits to put the underutilized resources in an energy saving state such as the base station sleep mode. Further energy efficiency could be granted by reducing the transmission power of BSs when traffic is low: even if the potential saving is lower than the one experienced in the sleep mode case, this technique does not have any drawbacks in terms of cell coverage. As previously stated, the network scenario comprises a basic layer of macro base stations, providing the coverage of a certain area, and a second overlapped layer of micro base stations, providing the extra capacity in cell edge regions. This kind of deployment allows to obtain an energy saving solution based on the following degrees of freedom:

- Variable number of active micro BSs: the number of active micro BSs is adapted to the variations of the users' requested capacity. Since a uniform traffic is assumed, the BS candidate to be switched on or off can be randomly selected. Anyhow more sophisticated solutions are possible.

- Macro BS power adaptation: the transmission power of macro BSs is adjusted looking at the available network capacity and the amount of UEs requests. While offered capacity is greater (or lower) than requested capacity, transmission power can be decreased (or increased) by a fixed step (e.g. $1 d B$ ). The power transmitted by the macro BSs can be reduced down to the lower bound that guarantees the coverage of the macro cells. For example, considering a $-90 \mathrm{dBm}$ UE sensitivity and the Macro BS-UE pathloss model in Table I, $P_{T X}=2 W$ is a a reasonable lower bound value to not affect the coverage area.

The considered strategy [16] works as follows:

- Initialization stage: each macro BS takes a record of the traffic managed by itself and by the micro BSs within its coverage area, setting up the starting values of the deseasonalized, trend and seasonal H-W components as defined in [22]. The initialization stage must be at least one day.

- Adaptation stage: i) Traffic forecast: each hour the HW components are updated by taking into account the new traffic records and the forecast is computed at the beginning of each day by considering the last values of the H-W components. ii) Network adaptation: forecasted traffic is compared to the offered capacity calculated thanks to equation (1). So while network provides more capacity than the forecasted micro BS are switched off

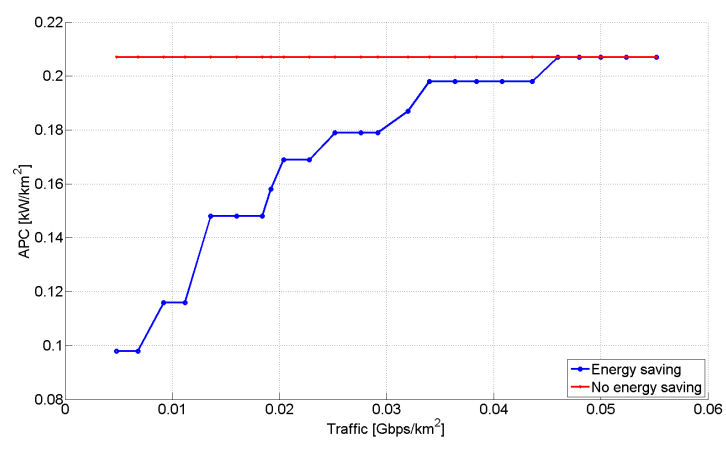

Fig. 2. Area power consumption

and, in case all micros are in sleeping mode, the power transmitted by macros is reduced. Likewise, while the provided capacity is lower than the forecast, the power transmitted by macros is increased and, in case all macro BSs transmit at maximum power, micro BSs are switched on.

\section{Simulation Results}

\section{A. Simulator and Metrics}

1) Simulator: The simulation set-up has been carried out in Matlab and NS-3 environments. Matlab is used for the traffic forecast, i.e., traffic generation, prediction and network adaptation, as described in Section III, while NS-3 is considered for the network performance assessment of the proposed energy saving solution. The salient features of the LTE/EPC simulation model based on NS-3 include the fully-implemented uplink and downlink LTE Radio Protocol stack functionalities, such as RRM algorithms, Inter-cell Interference Coordination, Load Balancing and Mobility Management. These features are extensively used in our simulation for modeling the network and testing the proposed approach. As for the Matlab simulator the inputs are the network deployment and the traffic variations during the considered period and the outputs are the traffic forecast, the number of active micro BSs and the transmission power of macro BSs. Regarding the NS-3 framework, for each simulated hour a certain number of UEs, depending on the generated traffic value, has been deployed randomly in the playground. Then the performance of the adapted scenario (Energy saving), that is the output of the Matlab simualtor, and the performance of the reference scenario (No energy saving, all micros active at the same time and all macros transmitting at maximum value) have been compared.

2) Metrics: The considered metrics for the results evaluation are: i) Area Power Consumption (APC) for the energy efficiency measurement of the proposed strategy with respect to the business as usual approach (APC is defined as: $A P C=P_{c, t o t} / S$, where $P_{c, t o t}$ is the total power consumption of all deployed macro and micro base stations and $S$ is the considered area); ii) the Throughput and the Delay per user for network performance analysis (the throughput is the amount of the effective received data in the time unit and the delay is the time between data transmission and reception). 


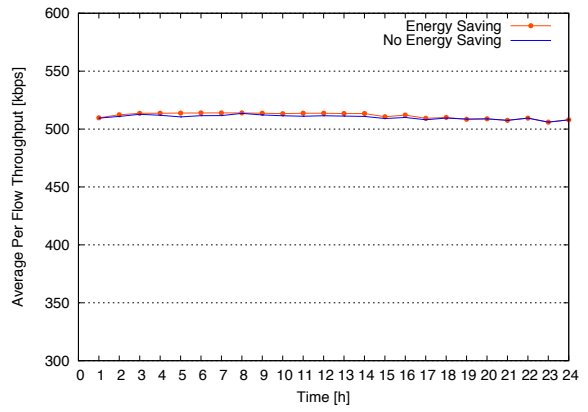

(a) Throughput

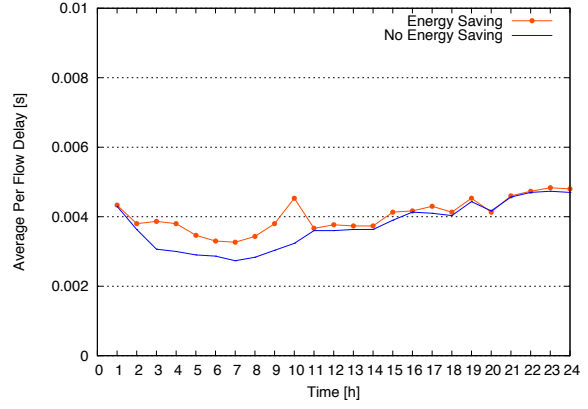

(b) Delay

Fig. 3. Average throughput and delay per user during the day for a cell

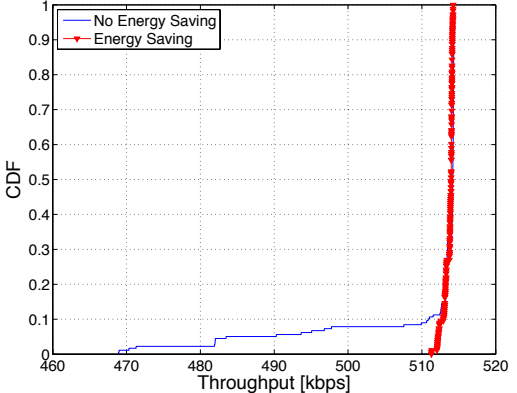

(a) Low traffic

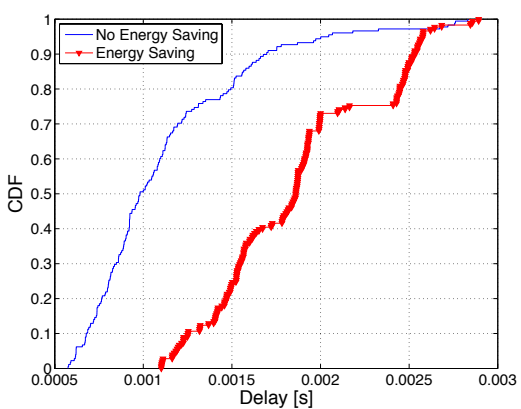

(a) Low traffic

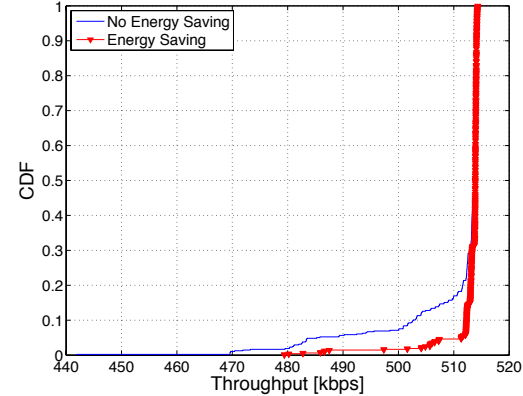

(b) Medium traffic

Fig. 4. User throughput CDF

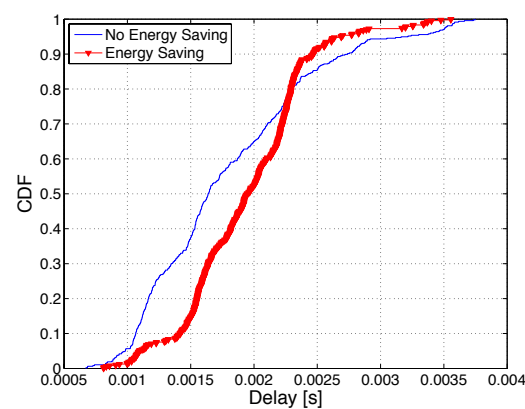

(b) Medium traffic

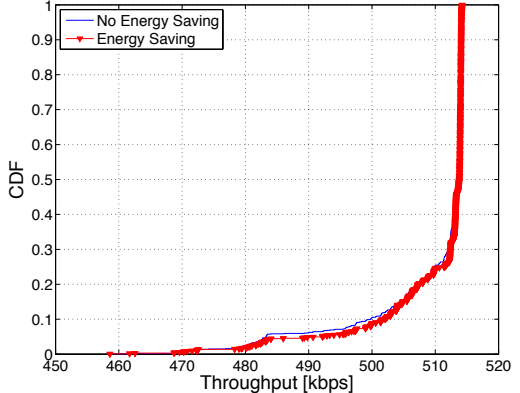

(c) High traffic

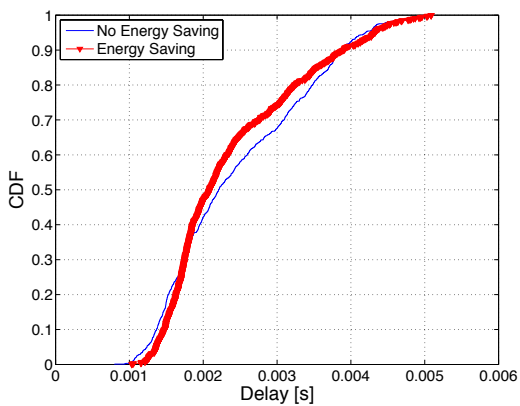

(c) High traffic

Fig. 5. User delay CDF

\section{B. Result Analysis}

The main effects of the proposed energy saving solution are shown in Fig. 2 where the area power consumption versus the measured traffic in the whole network is plotted. The chart shows the saving which is caused by the joint adaptation of micro base stations' activity and macro base stations' transmission power with respect to the reference case. In the reference case the APC results constant indipendently by the number of UEs in the network: this is because all the deployed macro and micro BSs are active at the same time, always transmitting at the maximum power. On the other hand, in the energy saving case, the APC is directly proportional to the traffic load, converging to the reference case when the network is heavily loaded. The performance of the proposed solution is presented in Fig. 3 where the average per user throughput and the average per user delay are plotted: these charts show that the implementation of the energy saving solution driven by the traffic forecast does not introduce any performance reduction for the served users. In fact, by adapting the radio resources, when the load of each cell is low, the users can still find a macro BS that can satisfy their requirements. In detail Fig. 3(a) reports the average per user values of the throughput which is experienced during the simulations: it is evident that the energy saving strategy results do not remarkably vary from the values obtained in the no energy saving case. On the other hand Fig. 3(b) shows that the average per flow delay 
is marginally affected by the implementation of the energy saving solution when the traffic load is lower, that is when the energy saving is higher. The main contribution affecting this performance metric is the retransmission at the base station side in case of low quality link. As a matter of fact, when some radio resources are not available for the users and the macro base station transmission power is reduced, the quality of each active radio link decreases and some users need more retransmissions with respect to the reference case, where users are connected to the closest macro or micro base station. However, this effect does not significantly reduce the user achieved quality of service in terms of throughput as shown in Fig. 3(a). A detailed evaluation of network performance is presented in Fig. 4 and Fig. 5 by considering low, medium and high traffic states. Network is in low traffic state during the hours when traffic is lower than the $30 \%$ with respect to the maximum value, medium during the hours when traffic is between $30 \%$ and $60 \%$, high when traffic is greater than $60 \%$. The user throughput CDFs are presented in Fig. 4. In particular Figure 4(a) shows the throughput distribution for low traffic values, Fig. 4(b) for medium traffic and Figure 4(c) for high traffic. It can be observed that when the energy saving strategy is implemented users experiment higher throughput gain in case of low and medium traffic. The reason is that in the reference case, when all radio resources are continuosly available, the interference between macro and micro BSs is higher than in the energy saving case. Similarly in Fig. 5 the delay performance for low (5(a)), medium (5(b)) and high traffic values $(5(\mathrm{c}))$ is presented. As previously stated, the throughput performance in the energy saving case is counterbalanced by higher delays of transmission with low and medium traffic, compared to the reference case. The user delay and throughput CDFs with high traffic, on the other hand, show a quite similar behaviour for both energy saving and no energy saving cases.

\section{CONCLUSION}

In this paper an energy saving solution for LTE heterogeneous networks is presented and evaluated. The energy saving is obtained by adapting the number of active micro base stations and the maximum transmission power of macro base stations to traffic variations. Since each cell needs the knowledge of traffic variations in its coverage area, a traffic forecast technique is used. Thanks to traffic forecast, the network is able to adapt itself to the capacity requested by users at different times of the day. In addiction a detailed analysis of system performance is done considering user throughput and delay. Results show the goodness of the forecasting technique and the energy saving introduced by the proposed solution. Moreover, the system evaluation shows a negligible reduction of user QoS with respect to the traditional no energy saving radio resource management.

\section{REFERENCES}

[1] O. Blume, D. Zeller, and U. Barth, "Approaches to Energy Efficient Wireless Access Networks," in 4th International Symposium on Com- munications, Control and Signal Processing (ISCCSP), May 2010, pp. $1-5$.

[2] A. Fehske, G. Fettweis, J. Malmodin, and G. Biczok, "The Global Footprint of Mobile Communications: The Ecological and Economic Perspective," IEEE Communications Magazine, pp. 55-62, Jul. 2011.

[3] C. Han, T. Harrold, S. Armour, S. Videv, P. M. Grant, H. Haas, J. S. Thompson, U. of Edinburgh Ivan Ku, C.-X. Wang, H.-W. U. T. A. Le, M. R. Nakhai, K. C. L. J. Zhang, and L. Hanzo, "Green Radio: Radio Techniques to Enable Energy-Efficient Wireless Networks," IEEE Communications Magazine, pp. 1-9, May 2011.

[4] T. Chen, Y. Yang, H. Zhang, H. Kim, and K. Honerman, "Network energy saving technologies for green wireless access networks," IEEE Wireless Communication, pp. 30-38, Oct. 2011.

[5] A. J. Fehske, F. Richter, and G. P. Fettweis, "Energy Efficiency Improvements through Micro Sites in Cellular Mobile Radio Networks," in IEEE GLOBECOM Workshops, Sep. 2009, pp. 1-5.

[6] S. Tombaz, A. Vastberg, and J. Zander, "Energy and Cost Efficient Ultra High Capacity Wireless Access," IEEE Wireless Communication, pp. 17, Oct. 2011.

[7] H. Klessig, A. J. Fehske, and G. P. Fettweis, "Energy Efficiency Gains in Interference-limited Heterogeneous Cellular Mobile Radio Networks with Random Micro Site Deployment," in 34th IEEE Sarnoff Symposium, May 2011, pp. 1-6.

[8] S. Morosi, A. Fanfani, and E. Del Re, "Network Deployment and RRM Strategies for Green Mobile Communications," in European Wireless 2012, Apr. 2012, pp. 1-6.

[9] G. Auer, "The EARTH Project: Towards Energy Efficient Wireless Networks," in Future Network \& Mobile Summit, Jun. 2010.

[10] M. Ajmone Marsan, L. Chiaraviglio, M. Meo, S. Buzzi, C. Guerrero, F. Idzikowski, Y. Ye, and J. L. Vizcaíno, "TREND: Toward Real Energy-efficient Network Design," in Sustainable Internet and ICT for Sustainability (SustainIT), Aug. 2012, pp. 1-6.

[11] G. Auer, I. Godor, L. Hevizi, M. A. Imran, J. Malmodin, P. Fazekas, G. Biczok, H. Holtkamp, D. Zeller, O. Blume, and R. Tafazolli, "Enablers for Energy Efficient Wireless Networks," in Proceedings of the IEEE 72nd Vehicular Technology Conference Fall (VTC 2010-Fall), Sep. 2010.

[12] M. Ajmone Marsan, L. Chiaraviglio, D. Ciullo, and M. Meo, "Optimal Energy Savings in Cellular Access Networks," in IEEE International Conference on Communication Workshop (ICC Workshop), Apr. 2009, pp. $1-5$.

[13] , "Multiple Daily Base Station Switch-Offs in Cellular Networks," in Fourth International Conference on Communications and Electronics (ICCE), Jul. 2012, pp. 1-6.

[14] G. Auer, V. Giannini, C. Desset, I. Godor, P. Skillermark, M. Olsson, M. A. Imran, D. Sabella, M. J. Gonzalez, O. Blume, and A. Fehske, "How Much Energy is Needed to Run a Wireless Network?" IEEE Wireless Communications, pp. 1-10, Oct. 2011.

[15] G. Auer, V. Giannini, I. GODOR, P. Skillermark, M. Olsson, M. A. Imran, D. Sabella, M. J. Gonzalez, C. Desset, and O. Blume, "Cellular Energy Efficiency Evaluation Framework," in IEEE 73rd Vehicular Technology Conference (VTC Spring), Feb. 2011, pp. 1-6.

[16] S. Morosi, P. Piunti, and E. Del Re, "Improving Cellular Network Energy Efficiency by Joint Management of Sleep Mode and Transmission Power," in Proceedings of the 24th Tyrrhenian Workshop on Digital Communications - Green ICT (TIWDC), Sep. 2013, pp. 1-6.

[17] Y. Wei, Z. Zhao, and H. Zhang, "Dynamic Energy Savings in Heterogeneous Cellular Networks Based on Traffic Prediction Using Compressive Sensing," in IEEE International Symposium on Communications and Information Technologies (ISCIT), Sep. 2011, pp. 1-6.

[18] S. Morosi, P. Piunti, and E. Del Re, "Sleep mode management in cellular networks: a traffic based technique enabling energy saving," Transactions on Emerging Telecommunications Technologies, pp. n/an/a, Feb. 2013.

[19] “3GPP TR 36.814 V9.0.0,” Tech. Rep., Apr. 2010.

[20] "EARTH WP2 D2.3," Tech. Rep., May 2012.

[21] F. Capozzi, G. Piro, L. A. Grieco, G. Boggia, and P. Camarda, "Downlink packet scheduling in lte cellular networks: key designs, issues and survey," IEEE Communications surveys and tutorials, pp. 1-23, Jun. 2012.

[22] D. Tikunov and T. Nishimura, "Traffic Prediction for Mobile Network using Holt-Winter's Exponential Smoothing," in 15th International COnference on Software, Telecommunications and Computer Networks (SoftCOM), Aug. 2007, pp. 1-5. 